\title{
A Persuasive Study on Burnout and Psychological Disorders among Medical Practitioners and Health Care Staff During Pandemic and Overload Work Environment
}

\author{
Dr.S. Horizan Prasanna Kumar ${ }^{\mathrm{a}}$, Dr.M. Alagesan ${ }^{\mathrm{b}}$, Dr.J. Karthikeyan ${ }^{\mathrm{c}}$ \\ aDepartment of English and Foreign Languages, SRM Institute of Science and Technology, Kattankulathur \\ Campus, Tamilnadu, India. E-mail: horizans@ srmist.edu.in \\ ${ }^{\text {b}}$ Department of English and Foreign Languages, SRM Institute of Science and Technology, Kattankulathur \\ Campus, Tamilnadu, India. E-mail: alagesam@srmist.edu.in \\ ${ }^{\mathrm{c} D e p a r t m e n t}$ of English, Vellore Institute of Technology, Vellore, India. \\ E-mail: jkarthikeyan@vit.ac.in
}

Article History: Received: 11 January 2021; Accepted: 27 February 2021; Published online: 5 April 2021

\begin{abstract}
Burnout syndrome in recent days has creeped among the medical practitioners and supportive staffs in hospitals and health care units from around the globe, due to the pandemic situation that provoked because of COVID-19. Incessant flow of patients round the clock shattered the energy level of physicians and nurses who are in this life saving exercise without even caring about their own lives. But treating patients continuously in critical care unit leads to emotional fatigue and in a long run leads to futility among the health care workers. Analysing the root cause among the medical practitioners by means of their lifestyle, socio-psychological status and their inbuilt aptitudes will let the hidden reasons behind this burnout. By means of continuous monitoring, motivation and training they can overcome this syndrome and make their lives more meaningful for this society as well as their self. This paper reveals the common and psychological problems obsessed by the physicians during at their work place, their personal and social life situations with reference to studies carried out in different parts of the world.
\end{abstract}

Keywords: Burnout, Psychological Disorders, Lifestyle, Medical Practitioners, Pandemic.

\section{Introduction}

The life of common people in these lockdown and life threating infectious days has taken routes into numerous anguishes and syndromes. Medical practitioners and health care staffs are set with deepest reverence than ever before for their unremitting help given to the public, invariable of their social and economic status and to stop them from death. Aslam, H.D., et.al., (2014) Stress among the doctors can be classified on five significant factors that include working environments, lack of sleep, assignment, role burden, and impracticable hassles of patients. Sleep deprivation has a significant impact on the doctor's stress, which could affect their work daily. The mental pressure can escalate more when there is a lack of responsibility for the workplace, job appreciation, inflexible management, and financial compensation. Therefore, it is important to note that this problem should be considered as a significant part of the phycological factor as stress is not common, which could directly or indirectly affect many individuals and groups of the sector's economic and social wellbeing.

Sun, J., et.al., (2020) The knowledge on the influence of social support in physician's psychological health and their association with demographic indicators are limited. The socially supported physicians faced less amplitude of PH alteration than the unsupported physicians since good social support enhanced positive emotional experience. Depression and anxiety scores of physicians with higher professional titles and education were less compared to the ones with lower titles and education. The possible reason for such psychological conditions in lower titled physician attributes to medical disputes in the working environment, insults, physical hurt, uncivilised behaviour from patients. Another reason is the professional efficiency of higher titled and educated physicians, as they have a stronger grasp of knowledge; they are more capable in solving the problems of patients. Downey, G.B., et.al.(2017) Reduction in the stigmatization of mental ill-health and preventing the management from unnecessary blame, establishing wide network to support and mentor will be beneficial for the trainee medical practitioners for better achievement. Miedema, B., et.al., (2017) Some physicians may face more risk of harassment and abuse than others, such as physicians working in emergency rooms, psychiatric emergency services, and after-hour services, even those on house calls, are more likely to experience hostile experiences. Effectively eliminated by implementing appropriate policies, curricula on dealing with abusive events, reduced workloads, no power structures and tackling systematic issues. It will pave the way for health workers to function in an environment that is intolerance to abuse and does not intentionally or unintentionally encourage such abusive conduct.

\section{Burnout Syndrome}

Brindley, P.G., (2017) Burnout is about mere emotional frustration, and may be unavoidable when it comes to end-of - life treatment for others. Burnout seems more than just lonely, and about a middle age crisis. Burnout may also be correlated with hopelessness, impotence and despair. Burnout is, however, extremely prevalent in 
caring occupations and in people who are well trained, self-motivated and drawn to stressful employment where there are strong threats and rewards. It would not be shocking considering that healthcare professionals are among the largest rate of self-reported burnout. All medical staff would acknowledge high risks of feeling it directly or seeing that in their co-workers. Screening and burnout counselling is a normal progression, appropriately. In reality, a burnout period may clearly represent a loss of tolerance which includes a break-up in partnership, unscrupulous health, lack of persistence. When endurance (i.e. a new partnership, fitness, time to retrain) may be recovered and burnout is quelled. Maintaining balance between work and life is a crucial method for revitalizing, maintaining focus and blow-off energy.

Hartzband, P. and Groopman, J., (2020) Burnout - a condition of "a motivation" is a major problem that erodes the quality and dignity of medical professionals. Most common reasons of physician burnout being, workload, inability to meet the demands of hospital authorities, officials and insurance companies, who seems to dominate over doctors and their profession. Several reforms have been made in the medical system such as implementation of EHR and use of performance metrics, which ended up in even complicated results along with imposing an extra financial burden to the medical sector, annually. Excercises that can provide physical relaxation and practices which render mental ease can help doctors. But the these measures will be futile if less focus is given to moral values and professional ethics. Intrinsic motivation should be valued over extrinsic motivational factors such as money and related rewards. The flawless dedication to their career and selfless service provided to the society must be the motto of every physician. They must lead a career-oriented life, build on the 3 pillars - Competance, Autonomy and Relatedness. The social co-operation brought about by the current pandemic situation must be sustained for the well-being of our society and its time to evaluate the reforms implemented in the health care sector to prevent the earlier status quo and to foresee the problems of physicians as well.

Ahmad, W, et.al (2018) study was carried out in Pakistan aiming to analyse the stressors producing burnout and how it influence the doctor and patient relationship. A sample of 515 doctors ( House officers \& PG residents) participated in the study which concluded a valid sample of 487. Convenience sampling technique was used in 6 hospitals of Lahore. Data was analyzed using correlation and linear regression method. CBI and PPOS were the measuring instruments. Scale for the study was measured with a questionnaire consisted of 30 item under the headings as psychological, personal stressors, lifestyle and work.CBI was the dependent variable and PPOS as predictor variable. Study found that House officers have high level of burnout compared to PG residents, also females compared to males. Low income, long working hours, lack of autonomy and efficacy are found to be important stressors. Smoking was a popular choice to break stress. These stressors only imply relation to power sharing of doctor and patient relationship and not with burnout and doctor patient relation. Addressing those stressors can lead to improved care delivery.

Mack, D.O. (2018) The issue of burnout and workplace stress among physicians have received increased research attention. Burnout syndrome has been defined in different stages. It has a emotional exhaustion stage, depersonalization, work detachment stage etc. The current study on "State Chapter Perspective on Burnout and Resiliency" focused on the physicians in new group who demonstrate considerable distinction from the averages. It is understood that physicians have higher rates of emotional exhaustion, depersonalization. And there is a $10 \%$ increase in burnout from 2011 to 2014 among physicians. It is also clear that physicians work for an additional ten hours when compared to physicians in US. According to the study the level of resilience is much greater among physicians. This study also implies that burnouts among physicians can also be due to extended administrative tasks and other frustration in doing management activities. This study also provide clues as research pursues future answers by picturizing the differences in the components of burnout syndrome rates of emotional exhaustion and depersonalization among physicians in different states who are new. Zantinge, E.M., et. al (2009) The Progress of the General Practitioners are not disturbed even after long hours of discussion with patients who are psychologically challenged and needs diagnosis. Rather it turns as an opportunity for them to understand the patients better and suggest them with appropriate care.

\section{Psychological Stress during COVID-19}

Preti, E., (2020) COVID-19 and was declared a global pandemic on $11^{\text {th }}$ March 2020. The epidemic has not only affected the human race worldwide but also crippled the psychological balance of the healthcare workers who are the frontline workers of these kinds of epidemics or pandemics. The healthcare workers slowly but steadily suffer from psychiatric symptoms and psychological distress and other mental health problems. WHO has recommended that rapid reviews of empirical evidence can help in the establishment of healthcare systems. This review has methods like search strategy and selection criteria, data extraction from various countries, type of epidemic or pandemic, assessment scales, etc. They have synthesized the data with defensive factors related to these psychological consequences and summarised risk. Further, there are psychological impacts like stress, infection-related worries, high level of emotional exhaustion as burnouts. Besides, neuroticism, dysfunctional attachment, maladaptive capacities were found as risk factors for mental health outcomes. Now, following this 
empirical evidence, we can propose for individual attention, proper training, adequate protective supplies, organizing online support services to lessen the negative psychological responses of healthcare workers.

Kalaitzaki, et.al. (2020) COVID-19 has left healthcare system all over the world scrambling. The threat of the pandemic has exerted immense pressure on healthcare personnel all over the world. Exhausting hours and lack of proper facilities along with taking care of patients has had a huge impact on the mental struggle of the healthcare workers. Several reports and studies have already highlighted the problem. The focus should shift towards making patient care a positive experience to the personnel. A salutogenic framework has to be created that focuses and enhances on factors that help people to cope up and achieve health on exposure to stressful conditions or stress. Studies have suggested that promoting resilience in caregivers can have appositive impact and avoid distress. A world-wide coordinated and cooperative approach is necessary in this regard. These should be done during the pandemic and intensified later. The pandemic should be used as an opportunity to learn.

Mijiritsky, E., et.al., (2020) The point of the study was to investigate the relationship between mental distress and subjective overload among dental specialists in various countries, and whether it is related to COVID-19related elements. A cross-sectional overview was led among 1302 dental specialists from China, India, Israel, Italy, and the UK, who rounded out demographic data, COVID-19-related factor questions, subjective overload, and mental distress scales. The discoveries demonstrated that the positive relationship between subjective overload and mental distress was distinctive among countries, recommending a higher pace of power in Italy contrasted with China, India, and Israel (UK had close significance with China and Israel). The collaboration variable of the subjective overload and mental distress was essentially connected with a specific country, with those people detailing fear of contracting COVID-19 from patients, the dread of their families contracting COVID-19, and accepting enough expert information concerning COVID-19. Given the above, dental specialists were found to have raised degrees of subject overload and mental distress, which contrasted among the countries, probably because of certain background issues such as social, cultural, and environmental components.

Shechter, A., et.al., (2020) A survey conducted on healthcare workers who were treating COVID-19 in New York City assessed various causes of distress, these were analyzed by a cross-sectional study by following up psychological screening, coping behaviors and wellness practices, COVID-19 stressors, sleep, loneliness, psychological screening, etc. From the results, it stated that fears of disease transmission, working with symptoms, patient deaths, lack of guidelines and control; agony about family and personal life are the major stressor for distress in health care workers. Among the healthcare workers, Nurses/AP providers are more prone to an acute stress disorder, depression, and anxiety as they have different responsibilities and spending more time with the patients. Qualitative reports from the listening sessions and interviews provided the causes of stress in the study. The main source of stress is due to lack of access to PPE and lack of testing, worry about family and personal life as they may easily transmit the virus, lack of support from the organization, less access to childcare support, limited information about medical care and communication. Governments, hospitals, and the community should take responsibility to take care of the health care workers.

Abbasi, J., (2020) This paper provides us the gist of information regarding the mental and physical stress which getting worsens day by day under the curtain of COVID 19 among our doctors, physicians, and healthcare workers. Being in the first line of contact with the infection, they are the most sufferers, and still, they are saving mankind risking their life and health. The increasing pressure of being lonely, depressed, suffocated, traumatized, and full of guilt, they are facing the main challenge even bigger and long-lasting than corona. Higher government officials and influential people should make strategic and operational organs in the society which help our healthcare workers dealing with exponentially multiplying state of stress and negativity. They should develop and devise scientific measures to save the very life of the life-saver.

Cao, J., (2020) The medical workers are facing an uncertain battle for human race against COVID-19 outbreak. Medical workers like doctors, nurses and technicians are working round the clock confront it head - on. During this outbreak they need to be recommended to ensure their mental health and wellbeing. Front-line workers mostly take care of COVID-19 patients they likely to experience psychological stress. They require help to burnt out these stresses. So, Department of Psychological medicine conducts a qualitative and quantitative assessment of medical workers about their emotions. Each person of Front-line workers continuously monitored like interview basis. The assessment done by 9 a.m. to 9 p.m. every day. This assessment contains quantitative questionnaires about their living conditions, work time, workload, medical security, diet and sleep, emotions, coping strategies, body discomfort and patient healthy conditions. After completing questionnaires individually assessed the medical workers psychology. After that report and suggestion have been submitted to concern authority. That report suggests that all medical workers facing uncertain high degree of stress and mental health difficulties. Overall, the emotional stress and mental health not highly evaluated but this psychological assessment may support and burnt out negative impact of the emergency.

Hynan, M.T., (2020) Author is trying to exemplify the sufferings of hospitals and ignorance towards perinatal staff services in this pandemic situation. When whole nation is in the discourse of shortage of medical equipment and in facilitating field hospitals, NICU (Neonatal Intensive Care Unit) workers and psychologists has been placed under non-essential list and have been pushed to adopt telemedicine. Top priority was given to physical 
health of care providers neglecting their mental health, though psychological struggles of medical staff, victims and survivors were described as heroics which initially buffered emotional damage. With due increase in death count related to pandemic the epidemiologist can estimate the possibilities of PTS (Post Traumatic Stress), anxiety disorders among the general public. In the first year of birth, $20-30 \%$ or more NICU parents are diagnosed by mental disorders. $60 \%$ of mothers and $47 \%$ of fathers coming from NICU suffers from posttraumatic stress. According to burnout survey nearly $26.7 \%$ of NICU staff of various hospitals exhibited emotional exhaustion and the range lies in between $7.9-42.9 \%$. Further this rate is going to increase exponentially throughout the globe. Hence recruiting mental health staff, appraising the confidence can help perinatal staff to tackle the pandemic efficiently.

Man, M.A., et.al(2020) The most deadly pandemic has affected the entire population regardless of their profession and this study is based on a survey conducted among health care workings working in covid departments and non covid departments. The disease perception levels, stress levels, emotional distress and coping strategies were evaluated with the help of questionnaires namely, the brief illness perception questionnaire (IPQ), perceived stress scale (PSS 10), profile of emotional distress (PDE), and cognitiveemotional coping questionnaire (CERQ). The results were interpreted with the help of psychologist and it is observed that a high level of stress is faced by health care workers initially, but there were no differences between the groups. The coping strategies analysed showed positive reappraisal and focus replanning were mostly used during the crisis. The more the people are problem focussed more is the panic and fear to contract the disease and they are mentally distressed. People are accepting the fact that covid needs time to settle and are moving forward.

Liu, C.H., et.al., (2020)This study highlighted the major psychological challenges currently faced during the initial weeks of COVID-19, the study has identified the factors that associated with the clinical levels of depression, anxiety and PTSD symptoms. Sample of participants were collected from different groups categories and the survey was online and High loneliness and low distress tolerance was consistently associated with the high levels of depression and anxiety, young adults may have been particularly distressed in managing the school and work responsibilities during the pandemic time. People with high resilience were not associated with anxiety and people having support from family were not associated with any mental health outcomes, loneliness is a risk factor for mental health and high levels of it was associated with depression during this severity of COVID. Asian-Americans were less likely to report high levels of mental health symptoms compared to whites and family cohesion has served as one of the important protective factor for mental health for non-US born participants especially.

Anmella, G., et.al.(2020) In this journal discussed about mental health of a front-line workers. During this pandemic they are severely facing mental health problems, insomnia, depression and stress. The front-line workers more prone to this because lack of information about precautions. For this they took case study of 42 years old women working as front-line worker. She admitted to hospital suffering with anxiety, insomnia etc. On general examination followed by general blood test and urine toxic screening test there is no abnormality but her signs are speech was scared, decreased attention span and interactions was non syntonic. Following 3 days treatment the signs disappear. This is due to normal medical treatment followed by family health care support and mental health treatment. This should be noted that almost $85 \%$ of front-line workers presenting a brief psychotic disorder will develop a potentially serious mental illness long term. During this pandemic health care workers should be monitored regularly in the aspect of mental health and should be given at most support to them, IF necessary specific treatment should be given.

\section{Lifestyle}

Velimirović, I., et.al., (2017) Mental health workers are required to obtain empowerment and building of coping strategies. Stress level is getting different from people based on their personal status as education level, relationship status, length of service and working environment. Based on education level of participants, higher level of educated people is always committed to work and experienced more. Regarding the status of personal relationship, who are engaged to marry and live in romantic relationship gave higher results and single participants gave lower results. Based on length of service of the participants, there are many differences in lack of acknowledgment and lack of control. Participants who work relatively in short period of five years and more than twenty-five years expect more appreciation for their efforts and dedication. Based on work time of participants, when overtime work happens in unexpected situation, the stress level got increased and affects their personal life. Based on gender and age of Participants, there are no difference in work stress level.

Anguiano, M.C.C., et.al(2019) General Physicians (GPs) assume a significant role in the physical consideration of patients with serious psychological instability, so the point of this study was to dissect the connections between GPs' sociodemographic status, work-related factors and their discernments about mental illness. A descriptive, cross-sectional examination was directed in the Clinical Management Unit of Mental Health of the Regional Hospital of Malaga (Spain). The qualified population involved all General Physicians working in the 13 primary care centres in the emergency clinic's catchment zone during the investigation time 
frame. General Physicians were met to gather information on their perspectives and knowledge on mental illness, psychiatry and the neighbourhood emotional wellness group, as well as their sociodemographic status, professional qualifications and experience. Bivariate investigation was carried out and145 GPs addressed the survey $(77 \%)$. ANOVA uncovered that the vast majority of the patient care centres with the best relationship with their psychological wellness group and best attitude to mental illness were in the Central Community Mental Health Unit, which operated a synergistic model of care. These outcomes showed that GPs who worked all the more intimately with their specialist psychological well-being group had a superior impression of their relationship with the mental illness community and less stigmatisation as to mental disease.

\section{Motivation \& Training}

Barattucci, M et.al., (2019) The work-related stresses is increases among the peoples mainly in the Health care professionals. And theirs directly impact is seen on the patients, their relation with the patients and caregivers. So the mindfulness is important for the HCPs to improve their psychology. The IARA training is mainly for the HCPs along with the experts, doctors like neurologist, psychologist etc. The training is of 6 months in that all the activities which help to overcome stress level, emotional difficulties and anxiety. In that training the analysis of SWOT (strength, weakness, opportunities, and threat analysis) is done. Result found that there is simultaneously decreased in stress level, emotional difficulties and anxiety compared to those who was not participate in meetings. Also helps in psycho synthesis with the positive imagination. There is no difference in stress level due to age, job or sex. Generally the HCPs have an idea about the perceived stress, anxiety and emotional regulation which improves the psychological things which ultimately helps to the patients. Such training improves the self-awareness, acceptance of emotions, attention and emotional regulation also the breathing skills not only in mentally weak persons but also in everyone.

Humphreys, J, et.al., (2001) reviewed on the prove base for restorative workforce maintenance in country and rural communities in Australia. It contends clearly for more significant clarity of focus in progressing and future inquire about on maintenance. A conceptual show is hypothesized that can be utilized as the premise for exploring numerous unanswered questions relating to how best to boost the maintenance of specialists in rural and remote communities. Clearly, assessment of the viability of retention incentives requires a sound information of the determinants of proficient and individual completion and the triggers that give the catalyst for migration. In expansion, the demonstrate offers the opportunity for way better assessing the particular impacts of open interventions, in the frame of motivations outlined to hold specialists in provincial and inaccessible communities. The information obtained from such research can health specialists have certainty in best to target and address restorative workforce retention issues that as of now contribute to the deficiency of professionals in rural areas.

Bell, J., et.al., (2018) The RNS role emerged due to declining number of GPs who has been traditionally providing 24-hour healthcare in rural communities. RNS is a registered nurse with advanced nursing skills enabling independent autonomic practice as a "practice/district/community nurse". The present study used a descriptive exploratory approach to know the core descriptors of the RNS. The study was completed in 2 phases. Phase-1 consisted of document content analysis of 5 position descriptions of an RNS with samples from 5 NZ District Health Boards. Phase-1 results were used for Phase- 2 which held interviews with 4 in-service RNSs in NZ. The findings were presented within the domains of direct comprehensive care, system support, education, research, professional development, stress management, lack of recognition etc. The study reveals that RNS role requires advanced knowledge for independent patient care. RNSs must have postgraduate education to develop the advanced skills (for assessment, diagnosis and treatment of patients). The roles of RNSs require to be expanded to overcome the hurdles to become a Nurse Practitioner (NP). Furthermore, RNS visibility in the healthcare system can be enhanced through professional leadership and collaborative inter-professional education.

Kpassagou, B.L. and Soedje, K.M.A., (2017). A qualitative study was conducted in the paediatric oncology department in a teaching hospitals at Lomé capital city of Togo to explore the emotional strains faced by the health practitioners and how they cope with their work related stresses. A semi structured individual interview was conducted with 21 participants regarding their work experience, work timings, equipment availability, and their relationship with team and hierarchy. These interview audio and notes were transcribed and research team developed coding to manage the transcribed data and accomplish a thematic analysis which explores the key issues emerging from the data. From the analysis, it was found that the health practitioners were into anger and helplessness when there is a lack of treatment and medical equipment to treat cancer affected children. Another finding on how they cope with the emotional stress on the death of children was mainly normalization and acceptance of death. These findings highlight the need to enhance the emotional coping skills of health practitioners during their professional training and the need for palliative care policies. Further research can be done in understanding the factors associated with different emotional reactions which will help to identify strategies for alleviating emotional strains and improve the quality of life led by health practitioners. 
Al-Qahtani, M.F. and Alsubaie, A.S.R., (2020) This study is conducted to determine the stress level for female students in health care programs at government university in Saudi Arabia. These stresses affect the quality of the learning throughout their academic period and also in health point of view. There are various reasons for stresses experienced by the students includes academic work load, examinations, tight time schedule, family background, economic status, anxiety, depression, low academic grades. The Medical Student Stressor Questionnaire techniques are used to collect the data from 260 undergraduate students includes second, third and fourth year studying in six health care programs. The study was conducted during the month of March and April (2019-2020) to determine the natural stress levels of the students. This study reported that academic activities and group related activities are the main causes for higher stress level in students. Second and third year students showed a low stress level when compared with fourth year students. Therefore the stressed students should be identified in earlier stage and counselling can be done to sort out their problems in order to enhance the quality of education throughout the academic period. However this study is limited to female medical students and it cannot be formulated as common data.

\section{Conclusion}

Medical practitioners and health care staff suffered to the core during the current pandemic days with overloaded work environment. Though they were trained during their course of their education to face such environments, continuous flow of patients and incessant working hours penalized their patients and busted out and psychological outburst either in their work place or at their home. Increasing the number of such practitioners and assistance is the only remedy to overcome this issue as they too are humans with the same emotions and feelings. Government and Private entities should create policies pertaining to the welfare of these resources who are the fore runners in life saving.

\section{References}

1. Aslam, H.D., Jamil, R., \& Tariq, A. (2014). Stress of medical practitioners in private healthcare industry. Asian Social Science, 10(22), 111-116.

2. Sun, J., Sun, R., Jiang, Y., Chen, X., Li, Z., Ma, Z., Wei, J., He, C., \& Zhang, L. (2020). The relationship between psychological health and social support: Evidence from physicians in China. Plos one, 15(1), e0228152.

3. Downey, G.B., McDonald, J., \& Downey, R.G. (2017). The welfare of anaesthesia trainees survey. Anaesthesia and intensive care, 45(1), 73-78.

4. Miedema, B., MacIntyre, L., Tatemichi, S., Lambert-Lanning, A., Lemire, F., Manca, D., \& Ramsden, V. (2012). How the medical culture contributes to coworker-perpetrated harassment and abuse of family physicians. The Annals of Family Medicine, 10(2), 111-117.

5. Brindley, P.G. (2017). Psychological burnout and the intensive care practitioner: A practical and candid review for those who care. Journal of the Intensive Care Society. https://doi.org/10.1177/1751143717713088

6. Hartzband, P., and Groopman, J. (2020). Physician burnout, interrupted. New England Journal of Medicine.

7. Ahmad, W., Ashraf, H., Talat, A., Khan, A.A., Baig, A.A., Zia, I., Sarfraz, Z., Sajid, H., Tahir, M., Sadiq, U., \& Imtiaz, H. (2018). Association of burnout with doctor-patient relationship and common stressors among postgraduate trainees and house officers in Lahore-a cross-sectional study. Peer J, 6, e5519.

8. Mack, D.O. (2018). A state chapter perspective on burnout and resiliency. The Journal of the American Board of Family Medicine, 31(1), 5-6.

9. Zantinge, E.M., Verhaak, P.F., De Bakker, D.H., Van Der Meer, K., \& Bensing, J.M. (2009). Does burnout among doctors affect their involvement in patients' mental health problems? A study of videotaped consultations. BMC Family Practice, 10(1), 60.

10. Preti, E., Di Mattei, V., Perego, G., Ferrari, F., Mazzetti, M., Taranto, P., Di Pierro, R., Madeddu, F., \& Calati, R. (2020). The Psychological Impact of Epidemic and Pandemic Outbreaks on Healthcare Workers: Rapid Review of the Evidence. Current psychiatry reports, 22(8), 1-22.

11. Kalaitzaki, A.E., Tamiolaki, A., \& Rovithis, M. (2020). The healthcare professionals amidst COVID-19 pandemic: A perspective of resilience and posttraumatic growth. Asian Journal of Psychiatry, 52, $102172-102172$.

12. Mijiritsky, E., Hamama-Raz, Y., Liu, F., Datarkar, A.N., Mangani, L., Caplan, J., \& Shacham, M. (2020). Subjective Overload and Psychological Distress among Dentists during COVID19. International journal of environmental research and public health, 17(14), 5074. 
13. Shechter, A., Diaz, F., Moise, N., Anstey, D.E., Ye, S., Agarwal, S., \& Claassen, J. (2020). Psychological distress, coping behaviors, and preferences for support among New York healthcare workers during the COVID-19 pandemic. General hospital psychiatry, 66, 1-8.

14. Abbasi, J. (2020). Prioritizing Physician Mental Health as COVID-19 Marches On. Jama, 323(22): 2235-2236.

15. Al-Qahtani, M.F., \& Alsubaie, A.S.R. (2020). Investigating Stress and Sources of Stress among Female Health Profession in a Saudi University. Journal of Multidisciplinary Healthcare, 13, 477-484.

16. Cao, J., Wei, J., Zhu, H., Duan, Y., Geng, W., Hong, X., \& Zhu, B. (2020). A study of basic needs and psychological wellbeing of medical workers in the fever clinic of a tertiary general hospital in Beijing during the COVID-19 outbreak. Psychotherapy and psychosomatics, 1-3.

17. Hynan, M.T. (2020). Covid-19 and the need for perinatal mental health professionals: now more than ever before. Journal of Perinatology, 1-2.

18. Man, M.A., Toma, C., Motoc, N.S., Necrelescu, O.L., Bondor, C.I., Chis, A.F., Lesan, A., Pop, C.M., Todea, D.A., Dantes, E., \& Puiu, R. (2020). Disease Perception and Coping with Emotional Distress during COVID-19 Pandemic: A Survey among Medical Staff. International Journal of Environmental Research and Public Health, 17(13), 4899.

19. Liu, C.H., Zhang, E., Wong, G.T.F., \& Hyun, S. (2020). Factors associated with depression, anxiety, and PTSD symptomatology during the COVID-19 pandemic: Clinical implications for US young adult mental health. Psychiatry Research, 290, 113172.

20. Anmella, G., Fico, G., Roca, A., Gómez, M., Vázquez, M., Murru, A., Pacchiarotti, I., Verdolini, N., \& Vieta, E. (2020). Unravelling potential severe psychiatric repercussions on healthcare professionals during the COVID-19 crisis. Journal of Affective Disorders, 273: 422-424.

21. Velimirović, I., Vranko, M., Ferić, M., \& Jendričko, T. (2017). Burnout syndrome in mental health professionals: Psychiatric hospital setting. Alcoholism and psychiatry research: Journal on psychiatric research and addictions, 53(2), 123-138.

22. Anguiano, M.C.C., Aragón, A.B., Fernández, D.A. and Küstner, B.M. (2019). Perceptions about mental illness among general practitioners. International journal of mental health systems, 13(1), 27.

23. Barattucci, M., Padovan, A.M., Vitale, E., Rapisarda, V., Ramaci, T., \& De Giorgio, A. (2019). Mindfulness-Based IARA Model ${ }^{\circledR}$ Proves Effective to Reduce Stress and Anxiety in Health Care Professionals. A Six-Month Follow-Up Study. International journal of environmental research and public health, 16(22), 4421.

24. Humphreys, J., Jones, J., Jones, M., Hugo, G., Bamford, E., \& Taylor, D. (2001). A critical review of rural medical workforce retention in Australia. Australian Health Review, 24(4), 91-102.

25. Bell, J., Crawford, R. and Holloway, K. (2018). Core components of the rural nurse specialist role in New Zealand. Rural \& Remote Health, 18(2).

26. Kpassagou, B.L., \& Soedje, K.M.A. (2017). Health practitioners' emotional reactions to caring for hospitalized children in Lomé, Togo: a qualitative study. BMC Health Services Research, 17(2), 700.

27. Al-Qahtani, M.F., \& Alsubaie, A. S. R. (2020). Investigating Stress and Sources of Stress Among Female Health Profession Students in a Saudi University. Journal of Multidisciplinary Healthcare, 13, 477-484. 